\title{
Newton-Hooke algebras, nonrelativistic branes, and generalized pp-wave metrics
}

\author{
Jan Brugués \\ Departament ECM, Facultat de Física, Universitat de Barcelona, Diagonal 647, E-08028 Barcelona, Spain \\ Joaquim Gomis \\ Departament ECM, Facultat de Física, Universitat de Barcelona, CER for Astrophysics, Particle Physics and Cosmology, \\ Diagonal 647, E-08028 Barcelona, Spain, Centro de Estudios Científicos (CECS) Casilla 1469, Valdivia, Chile \\ Kiyoshi Kamimura \\ Department of Physics, Toho University, Funabashi 274-8510, Japan
}

(Received 15 March 2006; published 26 April 2006)

\begin{abstract}
The Newton-Hooke algebras in $d$ dimensions are constructed as contractions of dS(AdS) algebras. Nonrelativistic brane actions are WZ terms of these Newton-Hooke algebras. The NH algebras appear also as subalgebras of multitemporal relativistic conformal algebras, $S O(d+1, p+2)$. We construct generalizations of pp-wave metrics from these algebras.
\end{abstract}

DOI: 10.1103/PhysRevD.73.085011

PACS numbers: 11.10.Ef, 03.65.Fd, 11.25.- w, 45.20.D-

\section{INTRODUCTION}

In order to understand the AdS/CFT correspondence at string level one must consider sectors of string theory in which certain degrees of freedom decouple from the rest of degrees of freedom in suitable limits. Such decoupling sectors have different asymptotic symmetries compared to the full theory. The best known example is the BMN [1] sector of the string theory in $\operatorname{AdS}_{5} \times \mathrm{S}^{5}$. The relevant symmetry of the BMN sector is a super pp-wave algebra, which is a particular contraction of the $s u(2,2 \mid 4)$, see for example [2].

Nonrelativistic string theory in flat space [3], see also [4-6] is another example of a consistent decoupled sector of the bosonic string theory. The basic idea of the decoupling limit is to take a particular nonrelativistic limit such that only states satisfying a Galilean invariant dispersion relation have finite energy, while the rest decouple. This limit has been extended to other relativistic supersymmetric string theories [7,8]. In particular for the case of $\mathrm{AdS}_{5} \times \mathrm{S}^{5}$ we have found the nonrelativistic string theory in a suitable gauge reduces to a supersymmetric free field theory in $\mathrm{AdS}_{2}$.

Here we will construct nonrelativistic bosonic algebras by contracting $\mathrm{AdS}(\mathrm{dS})$ algebras. They are generalization of the Newton-Hooke $(\mathrm{NH})$ algebras $[9,10]$ to the brane systems. We will apply the nonlinear realization to these algebras and we will see how it is possible to construct the nonrelativistic brane actions as WZ terms. This property is due to the fact that one can construct a closed and invariant $p+2$ form but the associated $p+1$ form cannot be written in terms of left invariant forms. The $p+2$ EilenbergChevalley cohomology is nontrivial.

We will also see how the NH algebra can be extended with noncentral charges. It is due to an existence of nontrivial vector valued 2 form on a representation of the stability group.
We will also construct generalization of pp-wave metrics from these extended algebras. For the case of the ordinary $\mathrm{NH}$ algebra we construct the pp-wave metric [11] and its generalization with angular momentum, the gyraton [12]. For the NH brane algebras we will see these metrics live in a space with more than one timelike direction. Related to this point we will also show that the brane $\mathrm{NH}$ algebras in $d$ dimensions are subalgebras of multitemporal conformal algebras, $S O(d+1, p+3)$.

The organization of the paper is as follows. In Sec. II we study the brane NH algebras. In Sec. III we construct the actions as WZ terms. In Sec. IV we construct the noncentral extended NH algebras. In Sec. V we construct the generalized pp-wave metrics from these algebras and finally in Sec. VI we see the algebras are subalgebras of multitemporal conformal algebras.

\section{NEWTON-HOOKE ALGEBRAS}

The particle Newton-Hooke algebra $\mathrm{NH}_{+(-)}$in $d$ dimensions is obtained by contraction of $\mathrm{dS}(\mathrm{AdS})$ algebra in a similar manner as the Galilei algebra is obtained by contraction of Poincare algebra [9]. The dS(AdS) algebra is

$$
\begin{aligned}
{\left[M_{m n}, M_{r s}\right] } & =i\left(\eta_{m r} M_{n s}+\eta_{n s} M_{m r}-\eta_{m s} M_{n r}-\eta_{n r} M_{m s}\right) \\
{\left[M_{m n}, P_{r}\right] } & =i\left(\eta_{m r} P_{n}-\eta_{n r} P_{m}\right) \\
{\left[P_{m}, P_{n}\right] } & = \pm \frac{i}{R^{2}} M_{m n},
\end{aligned}
$$

where $m=0,1, \ldots, d-1, \eta_{m n}=(-;+\ldots+), \pm$ in the last commutator corresponds to $\mathrm{dS}(\mathrm{AdS})$ and $R$ is the radius of the $\mathrm{dS}(\mathrm{AdS})$ space. It is the isometry group of the homogeneous space with a constant curvature $\pm R^{-2}$. It is embedded in a $d+1$ dimensional space of coordinates $u^{M},(M=0,1, \ldots, d-1, d)$ with a constraint

$$
\eta_{m n} u^{m} u^{n}+\eta_{d d}\left(u^{d}\right)^{2}=\eta_{d d} R^{2},
$$


where $\eta_{M N}=\left(-;+\ldots+, \eta_{d d}\right)$ and $\eta_{d d}=+1$ for the $\mathrm{dS}$ and $\eta_{d d}=-1$ for AdS space.

In this paper we study the Newton-Hooke algebras associated with a class of nonrelativistic $p+1$ dimensional objects ( $p$-branes) living in the $d$ dimensional space-time, that we call $\mathrm{NH}$ brane algebras and are also denoted by $\mathrm{NH}_{ \pm}$. These algebras are obtained by a suitable contraction of the $d$ dimensional $\mathrm{dS}(\mathrm{AdS})$ algebra. The contraction is done by performing the following scaling on the generators

$$
\begin{gathered}
P_{\mu}=\frac{1}{\omega} \tilde{P}_{\mu}, \quad \mu=0,1, \ldots, p, \quad P_{a}=\tilde{P}_{a}, \\
a=p+1, \ldots, d-1, \quad M_{\mu \nu}=\tilde{M}_{\mu \nu} \\
M_{\mu b}=\omega \tilde{M}_{\mu b} \quad M_{a b}=\tilde{M}_{a b} \quad R=\omega \tilde{R},
\end{gathered}
$$

where $\omega$ is a dimensionless parameter, and we take $\omega \rightarrow$ $\infty$. The Greek indices denote the longitudinal coordinates $(\mu=\{0, \ldots, p\})$ to the brane whereas Latin indices are the transverse coordinates $(a=\{p+1, \ldots, d-1\})$ to the brane. This scaling is suggested by the nonrelativistic limit of relativistic branes [3-8]. We get for the nonzero commutators [13]

$$
\begin{aligned}
& {\left[P_{\mu}, P_{\nu}\right]=} \pm \frac{i}{R^{2}} M_{\mu \nu}, \\
& {\left[M_{\mu \nu}, P_{\rho}\right]=} i\left(\eta_{\mu \rho} P_{\nu}-\eta_{\nu \rho} P_{\mu}\right), \\
& {\left[M_{\mu \nu}, M_{\rho \sigma}\right]=} i\left(\eta_{\mu \rho} M_{\nu \sigma}+\eta_{\nu \sigma} M_{\mu \rho}-\eta_{\mu \sigma} M_{\nu \rho}\right. \\
&\left.-\eta_{\nu \rho} M_{\mu \sigma}\right), \\
& {\left[M_{a b}, P_{c}\right]=} i\left(\eta_{a c} P_{b}-\eta_{b c} P_{a}\right), \\
& {\left[M_{a b}, M_{c d}\right]=} i\left(\eta_{a c} M_{b d}+\eta_{b d} M_{a c}-\eta_{a d} M_{b c}-\eta_{b c} M_{a d}\right), \\
& {\left[P_{\mu}, P_{a}\right]= \pm \frac{i}{R^{2}} M_{\mu a}, } \\
& {\left[M_{\mu a}, P_{\nu}\right]=i \eta_{\mu \nu} P_{a}, } \\
& {\left[M_{\mu \nu}, M_{\rho c}\right]=i\left(\eta_{\mu \rho} M_{\nu c}-\eta_{\nu \rho} M_{\mu c}\right), } \\
& {\left[M_{\mu a}, M_{b c}\right]=-i\left(\eta_{a b} M_{\mu c}-\eta_{a c} M_{\mu b}\right) . }
\end{aligned}
$$

The generators $P_{\mu}$ and $M_{\mu \nu}$ form a $p+1$ dimensional dS(AdS) subalgebra (4) while the $P_{a}$ and $M_{a b}$ form a $d-$ $p-1$ dimensional Euclidean subalgebra (5). If we further take the limit $R \rightarrow \infty$ we obtain the nonrelativistic Galilean brane algebras [6].

\section{NH BRANE ACTIONS FROM NON-LINEAR REALIZATIONS}

Let us consider the coset $\mathrm{NH}_{ \pm} / H$ with the stability group $H=\left\{M_{\mu \nu}, M_{a b}\right\}$. We parametrize the coset element as

$$
g=g_{0} e^{i y^{a} P_{a}} e^{i v^{\mu a} M_{\mu a}} .
$$

$g_{0}$ is an element of $\mathrm{dS}_{p+1}\left(\mathrm{AdS}_{p+1}\right)$ and the corresponding Maurer-Cartan one form is

$$
\Omega_{0}=-i g_{0}^{-1} d g_{0}=e^{\mu} P_{\mu}+\frac{1}{2} w^{\mu \nu} M_{\mu \nu}
$$

where $e^{\mu}$ and $w^{\mu \nu}$ are $\mathrm{dS}_{p+1}\left(\mathrm{AdS}_{p+1}\right)$ vielbein and spin connection, the explicit forms depend on the parametrization of $g_{0}$. The Maurer-Cartan one form $\Omega_{0}$ verifies the Maurer-Cartan equation $d \Omega_{0}+i \Omega_{0} \wedge \Omega_{0}=0$. In components it is

$$
d e^{\mu}+w_{\nu}^{\mu} e^{\nu}=0, \quad d w^{\mu \nu}+w_{\rho}^{\mu} w^{\rho \nu}= \pm \frac{1}{R^{2}} e^{\mu} e^{\nu} .
$$

The second equation shows that the longitudinal space has constant curvature $\pm R^{-2}$.

The total Maurer-Cartan one form, $\Omega$ is

$$
\begin{aligned}
\Omega= & -i g^{-1} d g=L^{\mu} P_{\mu}+L^{a} P_{a}+L^{\mu a} M_{\mu a}+\frac{1}{2} L^{\mu \nu} M_{\mu \nu} \\
& +\frac{1}{2} L^{a b} M_{a b}
\end{aligned}
$$

where [14]

$$
\begin{array}{clrl}
L^{\mu}=e^{\mu}, & L^{a}=d y^{a}+e^{\mu} v_{\mu}^{a}, & L^{\mu \nu}=w^{\mu \nu}, \\
L^{\mu a}=d v^{\mu a}+w^{\mu \nu} v_{\nu}{ }^{a} \mp e^{\mu} \frac{y^{a}}{R^{2}}, & L^{a b}=0 .
\end{array}
$$

These left invariant forms verify the Maurer-Cartan equation $d \Omega+i \Omega \wedge \Omega=0$, more explicitly,

$$
\begin{aligned}
d L^{\mu}+L^{\rho} L_{\rho}{ }^{\mu} & =0, \\
d L^{a}+L^{\rho} L_{\rho}{ }^{a}+L^{c} L_{c}{ }^{a} & =0, \\
d L^{\mu \nu}+L^{\mu \rho} L_{\rho}{ }^{\nu} \mp \frac{1}{R^{2}} L^{\mu} L^{\nu} & =0, \\
d L^{\mu a}+L^{\mu \rho} L_{\rho}{ }^{a}+L^{\mu c} L_{c}{ }^{a} \mp \frac{1}{R^{2}} L^{\mu} L^{a} & =0, \\
d L^{a b}+L^{a c} L_{c}{ }^{b} & =0 .
\end{aligned}
$$

We can construct the nonrelativistic Newton-Hooke brane action as a WZ term of the NH algebra. There is a closed $p+2$ form invariant under $H$ using the MC one form $L$ 's in (11) [15]

$$
\Omega_{p+2}=\frac{(-1)^{p+1}}{p !} \epsilon_{\mu_{0} \mu_{1}, \ldots, \mu_{p}} L^{\mu_{0}} \ldots L^{\mu_{p-1}} L^{\mu_{p} a} L_{a} .
$$

The $H$ invariance is manifest and the closure is shown by using the Maurer-Cartan Eqs. (12). There exists a $p+1$ form $\Omega_{p+1}$ such that $\Omega_{p+2}=d \Omega_{p+1}$, 


$$
\begin{aligned}
\Omega_{p+1}= & -\frac{\epsilon_{\mu_{0} \mu_{1}, \ldots, \mu_{p}}}{p !} e^{\mu_{0}} \ldots e^{\mu_{p-1}}\left[v^{\mu_{p}}{ }_{a} d y^{a}\right. \\
& \left.+e^{\mu_{p}}\left(\frac{v_{\nu a} v^{\nu a}}{2(p+1)} \pm \frac{y_{a} y^{a}}{2 R^{2}}\right)\right] .
\end{aligned}
$$

The $p+1$ form (14) is not expressible in terms of the left invariant one form $L$ 's in (11). Therefore we have a nontrivial $p+2$ cohomology group in the sense of EilenbergChevalley.

By taking the pullback of the $p+1$ form (14) on the world sheet;

$$
S_{\mathrm{WZ}} \equiv T \int \Omega_{p+1}^{*}=T \int d^{p+1} \xi \mathcal{L},
$$

where $T$ is the nonrelativistic $p$-brane tension and $\xi^{j}(j=$ $0,1, \ldots, p)$ are the parameters of the worldvolume. $\Omega_{p+1}^{*}$ is given by

$$
\begin{aligned}
\Omega_{p+1}^{*} & =e\left[v^{\mu}{ }_{a} e_{\mu}{ }^{j} \partial_{j} y^{a}+\left(\frac{v_{\nu a} v^{\nu a}}{2} \pm \frac{(p+1)}{2 R^{2}} y_{a} y^{a}\right)\right] d^{p+1} \xi \\
& \equiv \mathcal{L} d^{p+1} \xi
\end{aligned}
$$

where $e_{\mu}{ }^{j}$ is the inverse of the $p+1$-bein $e_{j}{ }^{\mu}$ and $e=$ $\operatorname{det}\left(e_{j}{ }^{\mu}\right)$. Since $v^{\mu a}$ are nondynamical Goldstone fields we can express them using their equations of motion as

$$
v_{\mu a}+e_{\mu}^{j} \partial_{j} y_{a}=0,
$$

which are equivalent to impose $H$ invariant constraints

$$
L^{a}=0,
$$

in other words the inverse Higgs mechanism [16].

In terms of the dynamical variables $y^{a}$ and $x^{\mu}$ the Lagrangian becomes

$$
\mathcal{L}=\frac{\sqrt{-g}}{2}\left[-g^{i j} \partial_{i} y^{a} \partial_{j} y_{a} \pm \frac{(p+1)}{R^{2}} y_{a} y^{a}\right],
$$

where $g_{i j}$ is the induced metric of the longitudinal space $\mathrm{dS}_{p+1}\left(\mathrm{AdS}_{p+1}\right)$

$$
\begin{aligned}
& g_{i j}=\eta_{\mu \nu} e_{i}{ }^{\mu} e_{j}{ }^{\nu}=\tilde{g}_{\mu \nu}(x) \partial_{i} x^{\mu} \partial_{j} x^{\nu}, \\
& g^{i j} g_{j k}=\delta^{i}{ }_{k}, \quad g=\operatorname{det} g_{i j}=-e^{2} .
\end{aligned}
$$

In the static gauge $x^{j}=\xi^{j}$ the Lagrangian describes a set of free scalar fields $y^{a}$ of mass $2=\mp \frac{p+1}{R^{2}}$ in the $\mathrm{d} \mathrm{S}_{p+1}\left(\mathrm{AdS}_{p+1}\right)$ space with the metric $\tilde{g}_{\mu \nu}(x)$.

In the limit $R \rightarrow \infty$ we recover the Galilean result [6] since in that case the dS(AdS) algebra (1) goes to the Poincare algebra and the Newton-Hooke algebra (4)-(6) becomes the Galilean algebra.

The Lagrangian (19) obtained here as a WZ term can also be obtained from the relativistic brane action in the $\mathrm{dS}(\mathrm{AdS})$ [17] by a nonrelativistic limit. In this limit the coordinates are rescaled as
$X^{\mu}=\omega x^{\mu}, \quad X^{a}=x^{a}, \quad R=\omega \tilde{R}, \quad T=\omega^{1-p} \tilde{T}$.

In the limit $\omega \rightarrow \infty$ of the Lagrangian there appears a divergent surface term but can be compensated by the presence of the $B$-field [3]. The resulting finite Lagrangian becomes one in (19).

\section{NON-CENTRAL EXTENSIONS OF NEWTON-HOOKE ALGEBRAS}

Here we want to study the extensions of the $\mathrm{NH}$ algebra (4)-(6). We look for closed 2-forms constructed from the left invariant one-forms (11) that transforms nontrivially under the stability group $H$, in particular, under $M_{\mu \nu}$ [19]. As we will see shortly these forms will be responsible for the existence of noncentral extensions of the $\mathrm{NH}$ algebra.

Let us consider a vector valued 2-form $L^{c} L_{c}{ }^{\mu}$ [21]. This form is not closed but we have

$$
d\left(L^{c}{L_{c}}^{\mu}\right) \sim-L^{\rho} L_{\rho}{ }^{c} L_{c}{ }^{\mu} .
$$

where $\sim$ means up to terms depending on $L^{\mu \nu}, L^{a b}$, in other words $L^{\mu \nu} \sim L^{a b} \sim 0$. If we introduce a new tensor valued one form $L_{z}^{\mu \nu}$ verifying

$$
d L_{z}^{\mu \nu}+L^{\mu c} L_{c}{ }^{\nu} \sim 0
$$

we can see that the 2 -form $L^{c} L_{c}{ }^{\mu}+L^{\rho} L_{z \rho}{ }^{\mu}$ is closed. This condition indicates locally the existence of a 1 -form $L_{z}^{\mu}$ such that

$$
d L_{z}^{\mu}+L^{c}{L_{c}}^{\mu}+L^{\rho} L_{z \rho}{ }^{\mu} \sim 0 .
$$

Now we take into account the tensor properties of $L_{z}{ }^{\mu \nu}$ and $L_{z}^{\mu}$ under $M_{\mu \nu}$. The Eqs. (23) and (24) become

$$
\begin{gathered}
d L_{z}^{\mu}+L^{\rho} L_{z \rho}{ }^{\mu}+L^{c} L_{c}{ }^{\mu}+L^{\mu \rho} L_{z \rho}=0, \\
d L_{z}^{\mu \nu}+L^{\mu c} L_{c}{ }^{\nu}+L^{\mu \rho} L_{z \rho}{ }^{\nu}+L_{z}{ }^{\mu \rho} L_{\rho}{ }^{\nu} \\
\mp \frac{1}{R^{2}}\left(L^{\mu} L_{z}{ }^{\nu}-L^{\nu} L_{z}{ }^{\mu}\right)=0
\end{gathered}
$$

that together with (12) define a free differential algebra.

If we introduce the generators $Z_{\mu}$ and $Z_{\mu \nu}$ dual to the forms $L_{z}^{\mu}$ and $L_{z}^{\mu \nu}$ we have the following commutation relations

$$
\left[P_{a}, M_{\nu b}\right]=i \eta_{a b} Z_{\nu}, \quad\left[M_{\mu a}, M_{\nu b}\right]=i \eta_{a b} Z_{\mu \nu}
$$

and

$$
\begin{gathered}
{\left[P_{\mu}, Z_{\nu}\right]= \pm i \frac{1}{R^{2}} Z_{\mu \nu}, \quad Z_{\mu \nu} \equiv-Z_{\nu \mu}} \\
{\left[P_{\mu}, Z_{\rho \sigma}\right]=-i \eta_{\mu[\rho} Z_{\sigma]},} \\
{\left[Z_{\mu}, M_{\rho \sigma}\right]=-i \eta_{\mu[\rho} Z_{\sigma]},} \\
{\left[Z_{\mu \nu}, M_{\rho \sigma}\right]=-i \eta_{\nu[\rho} Z_{\mu \sigma]}+i \eta_{\mu[\rho} Z_{\nu \sigma]} .}
\end{gathered}
$$


Notice we have a noncentral extension of the original Newton-Hooke algebra, in particular Z's do not commute with $P_{\mu}$, see Eq. (28). $L_{z}^{\mu}$ and $L_{z}^{\mu \nu}$ are left invariant Maurer-Cartan one forms of the above algebra if we introduce the group parameters $c^{\mu}$ and $c^{\mu \nu}$ with the generators $Z_{\mu}$ and $Z_{\mu \nu}$. They are

$$
\begin{aligned}
L_{z}^{\mu}= & d c^{\mu}+w^{\mu \nu} c_{\nu}+e^{\nu} c_{\nu}{ }^{\mu}-v^{\mu}{ }_{a} d y^{a}-\frac{1}{2} e^{\nu} v_{\nu a} v^{\mu a} \\
& \mp \frac{1}{2} e^{\mu} \frac{y^{a} y_{a}}{R^{2}}, \\
L_{z}^{\mu \nu}= & d c^{\mu \nu}+w^{[\mu \rho} c_{\rho}{ }^{\nu]} \mp \frac{e^{[\mu} c^{\nu]}}{2 R^{2}} \pm \frac{1}{2} e^{[\mu} v^{\nu] a} \frac{y_{a}}{R^{2}} \\
& +\frac{1}{2} v^{[\mu}{ }_{a} d v^{\nu] a} .
\end{aligned}
$$

At the level of the coset this implies to consider

$$
g=g_{0} e^{i y^{a} P_{a}} e^{i v^{\mu a} M_{\mu a}} e^{i c^{\mu} Z_{\mu}} e^{(i / 2) c^{\mu \nu} Z_{\mu \nu}}
$$

The noncentral algebra acts in a natural way in a bosonic "super" space $\left\{x^{\mu}, y^{a}, v^{\mu a}, c^{\mu}, c^{\mu \nu}\right\}$.

In Sec. III we have considered the unextended NH algebra and we have constructed an invariant closed $p+$ 2 form $\Omega_{p+2}$, (13) from $L$ 's. The potential $\Omega_{p+1}$ in (14) was not expressed in terms of $L$ 's and was cohomologically nontrivial. The resulting Lagrangian was WZ Lagrangian pseudo invariant under the $\mathrm{NH}$.

In the extended algebra instead we can construct an invariant $p+1$ form as

$$
\Omega_{p+1}^{\prime}=\frac{\epsilon_{\mu_{0} \mu_{1}, \ldots, \mu_{p}}}{p !} L^{\mu_{0}} \ldots L^{\mu_{p-1}} L_{z}^{\mu_{p}} .
$$

$\Omega_{p+1}^{\prime}$ is an invariant $p+1$ form and satisfies

$$
\Omega_{p+2}=d \Omega_{p+1}^{\prime}
$$

where $\Omega_{p+2}$ is given in (13). The $\Omega_{p+2}$ is cohomologically trivial in the extended algebra. $\Omega_{p+1}^{\prime}$ differs from $\Omega_{p+1}$ in (14) by a locally exact form

$$
\Omega_{p+1}^{\prime}=\Omega_{p+1}+d\left[\frac{(-1)^{p}}{p !} \epsilon_{\mu_{0}, \ldots, \mu_{p}} L^{\mu_{0}} \ldots L^{\mu_{p-1}} c^{\mu_{p}}\right] .
$$

The Lagrangian is the pullback of $\Omega_{p+1}^{\prime}$ which is the one associated with $\Omega_{p+1}$ plus a surface term proportional to $c^{\mu}$ and it is invariant under the extended $\mathrm{NH}$ algebra due to appropriate transformations of $c^{\mu}$.

\section{GENERALIZED PP-WAVE METRIC AND EXTENDED NH ALGEBRA}

Here we construct a nondegenerate relativistic metric from the generators of extended $\mathrm{NH}$ algebra by considering the dual forms (11) [22]

Let us first consider the case of the $\mathrm{NH}_{ \pm}$particle case $(p=0)$. We consider the quadratic $H$ invariant combina- tions of the generators

$$
C=-2 Z_{0} P_{0}+P_{a}^{2}+\frac{1}{R^{2}} M_{0 a}^{2} .
$$

The associated invariant metric is given by

$$
\begin{aligned}
d s^{2}= & \left(-2 d x^{0} d c^{0} \pm\left(d x^{0}\right)^{2} \frac{\left(y_{a}\right)^{2}}{R^{2}}+\left(d y_{a}\right)^{2}\right) \\
& +\left(d\left(R v^{0 a}\right) \mp d x^{0} \frac{y^{a}}{R}\right)^{2} .
\end{aligned}
$$

It is the metric of a pp-wave with angular momenta, the gyraton $[12,23,24]$, note that $x^{0}, c^{0}$ are lightlike coordinates and $\partial_{c}$ is a covariantly constant null Killing vector.

The ordinary pp-wave metric is obtained by considering the first term of (36). The relation among the pp-wave metric and the particle $\mathrm{NH}$ algebra was studied in $[11,25]$. This result agrees with the fact that the pp-wave algebra in $d+1$ dimensions is isomorphic to a central extended $\mathrm{NH}$ algebra in $d$ dimensions. Note that we have obtained a relativistic pp-wave metric from an extended nonrelativistic algebra.

In the case of the NH brane algebra the generalization of the pp-wave metric is obtained by considering the quadratic $H$ invariant combination of the generators

$$
C=2 \eta_{\mu \nu} P^{\mu} Z^{\nu}+P^{a} P^{a}
$$

the metric is

$$
d s^{2}=2 L_{\mu} L_{z}^{\mu}+L^{a} L^{a} .
$$

Using (11) and (30) we have

$$
d s^{2}=2 e_{\mu}(D c)^{\mu} \mp e_{\mu} e^{\mu} \frac{\left(y_{a}\right)^{2}}{R^{2}}+\left(d y_{a}\right)^{2},
$$

where $e^{\mu}, \omega^{\nu \rho}$ are $\mathrm{AdS}_{p+1}$ vielbein and spin connection, $(D c)^{\nu}=d c^{\nu}+\omega^{\nu \rho} c_{\rho}$ is the covariant derivative. Note that this metric contains $p+1$ covariantly constant null vectors $\partial_{c^{\mu}}$, therefore this metric lives in a space with more than 1 times.

\section{EXTENDED NH ALGEBRAS AS SUBALGEBRAS OF $S O(d+1, p+2)$}

The ordinary particle Galilei group in $d$ dimensions and its central extension, the Bargmann algebra, are a subgroup of Poincaré group in $d+1$ dimensions.

$$
(\text { Bargmann })_{d} \subset(\text { Poincare })_{d+1} \text {. }
$$

To see this fact we introduce the light cone indices \pm

$$
A_{ \pm} \equiv \frac{1}{\sqrt{2}}\left(A_{d} \pm A_{0}\right) .
$$

Their generators are identified with those of Poincare generators in $d+1$ dimension as 


$$
\begin{array}{cl}
H=P_{-}, & K_{a}=M_{+a}, \quad Z=P_{+}, \\
M_{a b}, & (a=1,2, \ldots d-1),
\end{array}
$$

where $H$ and $K_{a}$ are energy and boost generators in the Galilei algebra and $Z$ is the central charge.

For the particle $\mathrm{NH}$ algebras an analogous construction can be done where the role of the Poincare group is taken by the conformal group $S O(d+1,2)$. The group is linearly realized in $d+3$ dimensional space with metric $\eta_{M N}=$ $(-;+\ldots+,-),(M, N=\{0,1, \ldots, d, d+1, d+2\})$. It is useful to introduce two lightlike indices

$$
A_{ \pm} \equiv \frac{1}{\sqrt{2}}\left(A_{d} \pm A_{0}\right), \quad A_{ \pm^{\prime}} \equiv \frac{1}{\sqrt{2}}\left(A_{d+1} \pm A_{d+2}\right)
$$

so that the nonzero components of the metric are

$$
\begin{gathered}
\eta_{+-}=\eta_{+^{\prime}-^{\prime}}=1, \quad \eta_{a b}=\delta_{a b}, \\
(a, b=1, \ldots, d-1) .
\end{gathered}
$$

The $\mathrm{NH}$ algebras, $\mathrm{NH}_{ \pm}$, are subalgebras of $\operatorname{so}(d+1,2)$ whose generators are expressed in terms of $\operatorname{so}(d+1,2)$ generators $\mathcal{M}_{M N}$ as

$$
\begin{aligned}
& P_{0}=\frac{1}{R}\left(\mathcal{M}_{-^{\prime}+} \pm \mathcal{M}_{+^{\prime}-}\right), \quad P_{a}=\frac{1}{R} \mathcal{M}_{a+}, \\
& K_{a}=\mathcal{M}_{+^{\prime} a}, \quad M_{a b}=\mathcal{M}_{a b}, \quad Z=\frac{1}{R} \mathcal{M}_{+^{\prime}+} .
\end{aligned}
$$

In the case of the $\mathrm{NH}_{ \pm}$algebras for general $p$-branes we consider the "multitemporal" conformal group in $d+1+$ $p$ dimensions, i.e., $S O(d+1, p+2)$. We introduce $p+2$ sets of lightlike vectors $\left( \pm, \pm_{0}, \cdots, \pm_{p}\right)$ with $\eta_{+-}=$ $\eta_{+_{\mu}{ }_{\mu}}=1,(\mu=0, \ldots, p)$. The brane $\mathrm{NH}_{ \pm}$generators satisfying extended algebra (4)-(6) and (27)-(29) are given by

$$
\begin{array}{cc}
P_{\mu}=\frac{1}{\tilde{R}}\left(\mathcal{M}_{-_{\mu}+} \pm \mathcal{M}_{+_{\mu}-}\right), & P_{a}=\frac{1}{\tilde{R}} \mathcal{M}_{a+} \\
M_{\mu \nu}=\mathcal{M}_{+_{\mu}{ }^{-}}-\mathcal{M}_{+_{\nu}-}, & M_{\mu a}=\mathcal{M}_{+_{\mu} a}, \\
M_{a b}=\mathcal{M}_{a b}, \quad Z_{\mu}=\frac{1}{\tilde{R}} \mathcal{M}_{+_{\mu}+}, & Z_{\mu \nu}=\mathcal{M}_{+_{\mu}+_{\nu}} .
\end{array}
$$

Summing up we have shown that the extended $\mathrm{NH}$ groups for $p$-branes in $d$ dimensions are subgroups of multitemporal relativistic conformal groups in $d+p+1$ dimensions,

$$
(\text { extended } \mathrm{NH})_{d} \subset(S O(d+1, p+2))_{d+p+1} .
$$

\section{SUMMARY AND DISCUSSIONS}

We have constructed $\mathrm{NH}_{ \pm} p$-brane algebras in $d$ dimensions as contractions of dS(AdS) algebras. Nonrelativistic brane actions are constructed as WZ terms of these algebras since the $p+2$ Eilenberg-Chevalley cohomology group is nontrivial. These bosonic algebras have noncentral extensions due to the existence of a nontrivial vector valued 2 form on a representation of the stability group. These algebras appear also as subalgebras of a multitemporal relativistic conformal algebras, $S O(d+1, p+2)$.

Finally we have constructed a generalization of the ppwave metric from quadratic $H$ invariant combinations of the generators. For the case of NH brane algebras we have seen that these metrics lives in spaces with more than one time. Since extended NH algebras appear as an special limit of string theory, it will be interesting to see if one could give some physical meaning to these space with more than one time. It will be also interesting to see the relation with the two time physics, see for example [27].

\section{ACKNOWLEDGMENTS}

We acknowledge useful discussions with Eloy Beato, Glenn Barnich, Thom Curtright, Jaume Gomis, Marc Henneaux, Luca Mezincescu, Filippo Passerini, Tonie Van Proeyen, Jorge Russo, Paul Townsend, Peter West and Jorge Zanelli. Joaquim Gomis and Kiyoshi Kamimura acknowledge the Perimeter Institute for the hospitality, where a part of this work has been completed. This work is supported in part by MCYT FA 2004-04582C02-01, CIRIT 2005 SGR 00564 and MRTN-CT 2004005104.

\section{APPENDIX: METRICS FROM NONLINEAR REALIZATIONS}

Let us consider a spacetime group $G$ with an unbroken subgroup $H$. We split the Lie algebra generators $G$ into $G_{I} \in \mathcal{G}-\mathcal{H}$ and $G_{i} \in \mathcal{H}$, where $G_{I}$ is generator $\mathcal{G}$ which does not belong to the stability group $H$. We consider the coset $G / H$ with following parametrization

$$
g=e^{i G_{I} x^{I}}
$$

where $x^{I}$ represent all the Goldstone fields and $G_{I}$ all the broken generators.

The generators $G_{\hat{I}}$ of the whole group $G$ transform under the action of $G$ as the adjoint representation of the group

$$
\begin{aligned}
G_{\hat{I}} \rightarrow G_{\hat{I}}^{\prime} & =e^{-i \epsilon^{\hat{K}} G_{\hat{K}}} G_{\hat{I}} e^{i \epsilon^{\hat{K}} G_{\hat{K}}}=\Lambda_{\hat{I}}^{\hat{J}}(\epsilon) G_{\hat{J}}, \\
\Lambda_{\hat{I}}^{\hat{J}}(\epsilon) & =e^{\epsilon^{\hat{K}} f_{\hat{K} \hat{I}}^{\hat{I}}} ;
\end{aligned}
$$

where $\hat{I}=(I, i), \epsilon^{\hat{K}}$,s are the transformation parameters, $f_{\hat{K} \hat{I}}^{\hat{J}}$ are the structure constants.

The transformation of a coset element is

$$
g \rightarrow g^{\prime}=g_{0} g h^{-1}, \quad g_{0} \in G, \quad h \in H .
$$

where $h\left(g_{0}, x\right)$ is a compensating $H$ transformation and depends on $x$ as well as $g_{0}$ generally. The Maurer-Cartan one form transforms as

$$
\Omega=-i g^{-1} d g \rightarrow \Omega^{\prime}=h \Omega h^{-1}-i h d h^{-1} .
$$


which implies

$$
\Omega_{G / H}^{\prime}=h \Omega_{G / H} h^{-1} \quad \Omega_{H}^{\prime}=h \Omega_{H} h^{-1}-i h d h^{-1} .
$$

Taking into account that

$$
\Omega=-i g^{-1} d g=G_{I} L^{I}+G_{i} L^{i}
$$

we have

$$
\begin{aligned}
L^{\prime J} & =L^{I} \Lambda_{I}^{J}(\alpha) . \\
L^{\prime i} & =L^{I} \Lambda_{I}^{i}(\alpha)+L^{j} \Lambda_{j}{ }^{i}(\alpha)+\left(-i h d h^{-1}\right)^{i}
\end{aligned}
$$

where $\alpha \in H$ is the induced $H$ transformation and in general depends also on $g$.

Suppose we have a quadratic generator invariant under $H$ constructed from $G_{I}$ 's

$$
C=g^{I J} G_{I} G_{J} .
$$

The invariance of $C$ under $H$ means

$$
g^{I J} \Lambda_{I}^{K}(\alpha) \Lambda_{J}^{L}(\alpha)=g^{K L},
$$

and

$$
g^{I J} \Lambda_{I}{ }^{k}(\alpha) \Lambda_{J}{ }^{L}(\alpha)=0, \quad g^{I J} \Lambda_{I}{ }^{k}(\alpha) \Lambda_{J}^{\ell}(\alpha)=0
$$

for any $H$ transformation $\alpha^{i}$. If $g^{I J}$ is not singular the first of above equation means $\Lambda_{I}{ }^{k}(\alpha)=0$ and $H$ is the automorphism group. Note $C$ is not necessarily a Casimir operator of $G$. When the $g^{I J}$ in the (A8) is not singular (nondegenerate) we can construct $G$ invariant metric using its inverse; $g_{I J}$

$$
d s^{2}=g_{I J} L^{I} L^{J} .
$$

Actually it is invariant as

$$
g_{I J} L^{I} L^{J} \rightarrow g_{I J} L^{\prime I} L^{\prime J}=g_{I J} L^{K} \Lambda_{K}^{I} L^{L} \Lambda_{L}^{J}=g_{I J} L^{I} L^{J} .
$$

where we have used

$$
g^{K L} \Lambda_{K}^{I} \Lambda_{L}^{J}=g^{I J} \rightarrow g_{K L} \Lambda_{I}{ }^{K} \Lambda_{J}^{L}=g_{I J} .
$$

[1] D. Berenstein, J. M. Maldacena, and H. Nastase, J. High Energy Phys. 04 (2002) 013.

[2] M. Hatsuda, K. Kamimura, and M. Sakaguchi, Nucl. Phys. B632, 114 (2002).

[3] J. Gomis and H. Ooguri, J. Math. Phys. (N.Y.) 42, 3127 (2001).

[4] U. H. Danielsson, A. Guijosa, and M. Kruczenski, J. High Energy Phys. 10 (2000) 020.

[5] J. A. Garcia, A. Guijosa, and J.D. Vergara, Nucl. Phys. B630, 178 (2002).

[6] J. Brugues, T. Curtright, J. Gomis, and L. Mezincescu, Phys. Lett. B 594, 227 (2004).

[7] J. Gomis, K. Kamimura, and P. K. Townsend, J. High Energy Phys. 11 (2004) 051.

[8] J. Gomis, J. Gomis, and K. Kamimura, J. High Energy Phys. 12 (2005) 024.

[9] H. Bacry and J. M. Lévy-Leblond, J. Math. Phys. (N.Y.) 9, 1605 (1968).

[10] J. R. Derome and J. G. Dubois, IL Nuovo Cimento 9, 351 (1972).

[11] G. W. Gibbons and C. E. Patricot, Class. Quant. Grav. 20, 5225 (2003).

[12] V. P. Frolov, W. Israel, and A. Zelnikov, Phys. Rev. D 72, 084031 (2005).

[13] We will omit "tilde" on the rescaled generators hereafter.
[14] Recall that here and below in the text when \pm or $\mp$ appear, the upper indices correspond to $\mathrm{dS}$ and the lower indices to AdS.

[15] Our convention is $\epsilon^{01, \ldots, p}=-\epsilon_{01, \ldots, p}=+1$.

[16] E. A. Ivanov and V. I. Ogievetsky, Teor. Mat. Fiz. 25, 164 (1975).

[17] Actions of branes in AdS have constructed also using the nonlinear realization methods in [18].

[18] F. Delduc, E. Ivanov, and S. Krivonos, Phys. Lett. B 529, 233 (2002).

[19] For ordinary supersymmetry the role of Lorentz one-forms was considered in [20].

[20] V. Aldaya and J. A. de Azcarraga, J. Math. Phys. (N.Y.) 26, 1818 (1985).

[21] Other tensor valued two form like $L^{\rho} L_{\rho}{ }^{a}$ is exact and can be written in terms of invariant forms, $L^{\rho} L_{\rho}{ }^{a} \sim d L^{a}$.

[22] See the appendix for a general discussion

[23] A. Coley, R. Milson, V. Pravda, and A. Pravdova, Class. Quant. Grav. 21, 5519 (2004).

[24] We acknowledge Eloy Beato for discussions on this point

[25] Previous discussion along this direction is found in [26]

[26] C. Duval, G. W. Gibbons, and P. Horvathy, Phys. Rev. D 43, 3907 (1991).

[27] I. Bars, hep-th/9809034. 